\title{
Reinventing Staircases for Thermoplastic Additive Manufacturing
}

\author{
Mirko Daneluzzo $^{1(\otimes)}$ and Michele Daneluzzo ${ }^{2}$ \\ ${ }^{1}$ Dubai Institute of Design and Innovation, Ras Al Khor Road, Bldg 4 Dubai Design District, \\ Dubai, UAE \\ mirko.daneluzzo@didi.ae \\ 2 NYXO Visionary Design, In5 Design, Ras Al Khor Road, Bldg 4 Dubai Design District, \\ Dubai, UAE \\ michele@nyxostudio.com
}

\begin{abstract}
The paper presents an ongoing project focusing on the application of additive manufacturing technologies for the design of staircases. Additive digital fabrication allows architects to reinvestigate materials, processes, and creates new design opportunities to explore novel aesthetical and functional expression in architecture, enabling a reinterpretation of the typology of the staircase, using thermoplastic materials. This paper reviews the opportunities and challenges of using $3 \mathrm{D}$ printing for fabricating custom stairs with complex geometries in two studied configurations.
\end{abstract}

Keywords: 3D printing $\cdot$ Additive manufacturing $\cdot$ Staircase $\cdot$ Architectural components · PET-Carbon

\section{Additive Manufacturing in Architecture: From the Large-Scale of the Structure to the Medium-Scale of Interior Products}

The usage in architecture of additive manufacturing (AM) has shifted from producing scale modeling to a full-scale end-product, and it is usually referred to as large-scale 3D printing. As the word suggests, is a special type of AM that specializes in erecting largescale, heavy, and often permanent structures (Al Jassmi 2018). While these technologies have been successful for small-scale purposes, scaling up 3D printing for construction, and replacing conventional building methods, is yet a challenge (ibid.). The companies investing in the field focus their attention on the architectural envelope, so on how to build the main body of the building, usually incorporating structure and cladding. Although different printing technologies are using for example locally sourced clay like the Italian Company WASP, the state-of-the-art of large-scale AM in architecture is largely dominated by concrete type research efforts. Concrete is the world's most widely used engineered material (Ashby 2012), nevertheless, its low cost, its compressive performance, and its versatility made it a choice.

It is possible to identify two main approaches for the application of AM in architecture: the first one is about the in-situ fabrication of the whole building (the printable 
part of the building) in a continuous fashion, the second is about the in-factory prefabrication of components. Researchers at ETH Zurich are applying the latter approach for the design of staircases using the prefabrication of 3D-printed formwork for the manufacturing of custom concrete stairs (Jipa 2019). With the same logic of 3D printed formworks, the Dutch company Aectual, showcased a monolithic concrete staircase at the Dubai Design Week 2020. The projects analyzed in this paper operate in the same domain of prefabrication but suggest a different approach, where no formwork is used in the process of shaping the product. The numerically controlled fabrication process is used to materialize the digital model using only the material needed, embedding in a single body the architectural and the structural parts, without the need to cast other materials.

The research is under development as a project of Nyxo Studio, the practice cofounded by the authors. The project was initiated in 2019 to address alternative applications of 3D printing for architectural-scale purposes and involves the authors and Cristian $\mathrm{Li}$ Voi as assistant designer. The research practice of the authors is aimed at having a direct impact on the market, so these projects evaluate both the technical and economic feasibility. The projects illustrated in this paper are part of a series of reflections about the application of AM in architecture, shifting the attention from the large-scale approach to a medium-scale one. These medium-scale applications range between the scale of furniture and that of a room. It is believed by the authors that the exploration of this spectrum is just at the beginning, and it offers a large possibility of expansion for the near future.

\section{Fused Granular Fabrication for Medium-Scale Objects}

Large-scale robotic thermoplastic printing for manufacturing finite objects is still a young technology. The main products realized so far using these technologies are furniture pieces, like chairs and vases, printed with different thermoplastics like PLA, ABS, PETG, TPU, and PET. These projects are inspired by these applications in the furniture field. The staircase is indeed thought of, since the beginning, as a discrete object, where each unit has the size of a furniture piece. This analogy helped to channel the characteristics of the unit as a lightweight object, something mobile, easy to handle as a single piece. 3D printing is used to build the entire body of the module in an integrated way, to avoid any additional component. This analysis suggests avoiding using materials with a very high unit weight like concrete, but still capable of a decent tensile and compressive strength. The exploration of Dirk Van der Kooij with his Endless Chair (2010), is one of the first examples of the evolution of the Fused Deposition Modeling (FDM) prototyping technique into a fabrication scale, using thermoplastics in form of pellets. More commonly used in injection molding, pellets are not only less expensive but come in a wider range of materials than filament, including a long list of recycled and sustainable products. Pellet-based 3D printers can print large objects quicker than filament-based printers because a high volume of pellet material is fed into the extruder and thanks to a larger nozzle. It is usually referred to as Fused Granular Fabrication (FGF) and it is setting a standard in printing large objects using Thermoplastic materials. FGF is a very attractive and promising technology for applications in medium-scale architecture components, due to their speed, their capacity to print different materials, and availability 
on large scales. FGF is recently diffusing in the industry, also for the relatively low cost of the raw materials, that if we consider thermoplastic materials, they can run as low as $\$ 2.5$ per $\mathrm{kg}$.

FGF is available in two main configurations: gantry-based and robotic-arm-based. In the first case, an example is the printer MarkI or MarkII by the Belgian company Colossus. In the same category, we can consider the Delta 3MT by the Italian company WASP. Even if the Delta printer is equipped with a three arms stabilization system, and the degree of freedom of the machine is potentially higher than the gantry-based one, the printing process is the same, characterized by a horizontal stratification of the layers.

In the family of robotic-arm printers, there are companies like the UK-based Ai Built, The Spanish Nagami, the Dutch Aectuel, and the American company Branch Technology. The advantage of working with a robotic arm is that there is the possibility to print with the Tangential Continuity Method (TCM) (Al Jassmi 2018) that allows to overcome the horizontal layering and also concedes the possibility to print on pre-defined molds like in the case of Zaha Hadid's Bow Chair and The Rise Chair manufactured by Nagami. Another approach that the robotic-arm technique offers is the definition of spatial 3D lattice structures. Both cases make use of the increased degrees of freedom of a 6-axis robotic arm to generate a building path that could go beyond the horizontal stratification of a 3D axis machine.

For the project discussed in this paper, the choice fell on a gantry-based system for the relatively lower and easier operational costs respect a robotic-arm printing system, considering also the possibility for a hypothetical company to invest in the acquisition of the machine.

\section{Why 3D Printing a Staircase?}

Non-standard stairs have an important role in architecture, but their complex details pose significant fabrication challenges. One of the preferred materials for custom stairs is steel, which can be shaped in different ways, but with high costs and a lot of components. 3D printing can unlock an entirely new vocabulary of shapes, previously unavailable with traditional systems, and materials like steel, wood, and concrete. Only a minimal amount of 3D-printed plastic is required to deliver a very thin, stable shell. Complex topologies can be achieved with less effort, such elements can optimize the structural performance or improve functional aspects, as well as introduce a radically different aesthetic.

The market of interior non-standard staircases, leaving aside the in-situ concrete stairs, goes in two main directions. On the one hand, there is the umbrella of bespokemade products, on the other hand, there is the family of industrial in-kit products. The first case is generally defined by a specific design that is adapted to the context in every single component, this is also one of the main factors that raise the prices of these products. The overall result is usually intended to be organic, coherent as a whole.

The in-kit products are instead meant to be mass-manufactured, to reduce the design and manufacturing costs. In this staircase typology, the adaptability is shifted from the design phase to the assembly. The interaction between the standardized components is designed to guarantee a range of configurations. This advantage has a side effect from the aesthetical perspective: it is difficult here to achieve an aesthetical result that seems 
designed for the specific case, usually, these products are manifesting this adaptation, with discontinuities.

AM could bridge the two typologies with an affordable on-demand customized fabrication, creating staircases that can adapt to a specific context without losing the overall aesthetic quality.

As indicated in the CSC Leading Edge Forum (2012), the advantages of using 3D printing for such a product for interiors and medium-scale architecture components can be listed as follows: affordable customization; allows the manufacture of more efficient designs; lighter, stronger, less assembly required; one machine, unlimited product lines; efficient use of raw materials (less waste); pay by weight; complexity is free; batches of one, created on demand; print at point of assembly/consumption; new supply chain and retail opportunities. In the same report, there are highlights that, there are still some areas in need of further development. In particular, the possibility of printing large volumes economically, expanding the range of printable materials, using multiple materials in the same printer, to improve durability and quality as a final result. All these benefits make it worth exploring AM technologies for real applications. Architects are offered the chance to reinvent architectural components and the ecology of materials of the built environment by exploring forms and processes deemed impractical or inconceivable before.

With innovative construction materials/methods and better decision-making systems, not only projects are getting smarter but also it is an opportunity to build our environment more sustainable (Beyhan 2018).

\section{Main Characteristics and Goals}

One of the first goals considered in the design process of the staircase, was the reduction of the components, to speed up and simplify the assembly operations, and as a direct consequence, the reduction of the suppliers' chain. A big problem with the in-kit staircases is indeed the large number of components that need specialized labor and a long time to assemble the final product. The staircase is indeed conceived as a discreet assembly with components not bigger than small furniture, to avoid logistic problems. The module is so incorporating riser, tread, and string in one single element. This logic makes the module lightweight, easy to transport, and quick to install. The connection between the components is guaranteed by standard mechanical hardware employable for every size or configuration. In synthesis, the characteristics used to drive the design process are: integrating the components (riser, tread, stringer); lightweight; easy assembly; quick manufacturing; adaptability to specific configurations (with an organic whole aesthetics).

For structural integrity and due to the use of FGF technology, the module, which is a single step, is described as an open volume, or a reverse vase, with a section that allows a continuous printing path. The step is then a hollow volume, where the mechanical stress is handled by twisted surfaces operating as struts due to their triangular configuration. The dynamic section of the single module is designed to optimize the printing time while guaranteeing the required mechanical strength. The adaptability is reached through the definition of a parametric model of the module. The adjustments of the module affect the production costs only in the quantity of material used. 


\section{BRep Stereotomy: Conceptualization of the module's Geometry}

The design process to define the geometry of the modules is inspired by the art of stereotomy in gothic stone spiral staircases. Instead of working with subtraction, AM requires a digital model with the specific articulation of the surfaces defining the volume. This is an example of extending the use of computer-aided design (CAD) from being a medium of representation to a media of design and manufacturing (Celani 2002). Future architects are expected to become robotic-aware, in other words, able to consider the robotic arm constraints for the design of a given building element (Al Jassmi 2018). In this spirit, the proposed design process shows that the understanding of the fabrication process with its limits and the material behavior is important to define the physical articulation of the object, as a synthesis between aesthetics and mechanical performance. This methodology is compatible with most CAD packages that describe geometric objects as a collection of individual surfaces that are joint along their edges. Boundary representations (BRep) are highly efficient and offer a lot of flexibility in terms of design. The combination of BRep design and 3Dprinting for the construction of hollow components reduces manufacturing-related resource inputs because it only requires the amount of material that ends up in the printing well without too many losses (Reeves 2008). In addition, it makes the component lightweight and therefore, easy to handle.

\section{Design Configuration 1: Oblique Interlocking}

The volume of the step is here defined by four main surfaces: the tread, the riser, and the two opposite surfaces generating a quadrangular shape in its section at the ends. The staircase slope is used to define the perpendicular surfaces of connection between the modules. This inclination consequently determines the riser and its opposite back face. The riser and the back face are articulated on the outer shell, so to obtain a rebated joint and using in addition chemical adhesives to bond the modules together (Fig. 1).
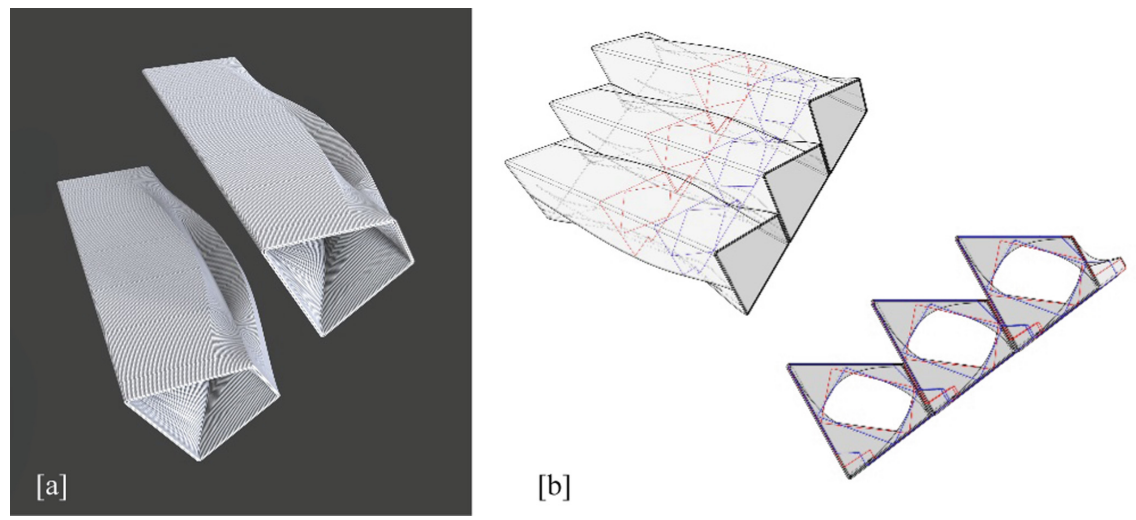

Fig. 1. Configuration 1, (a) rendering of two disjointed modules. (b) Diagrams showing the articulation of the inner and outer surface and relative areas of connection. 
The inner surface is designed as a twisted element that interpolates the lateral section into the middle one, exactly where the step needs structural reinforcement. The tread, for example, needs to be a large flat surface to guarantee comfortable usage. As a single thin layer of material subjected to the direct stress of the weight of the user, it needs reinforcements. The inner surface indeed articulates to distribute the loads through triangulations (Fig. 1b, Fig. 3a). The combination of the box-like outer shell with the closed triangulated geometry in section, and the twisted surface configuration, guarantees mechanical efficiency with thin layers, avoiding deformations due to compression and tensile forces, and torsions of the planar surfaces.
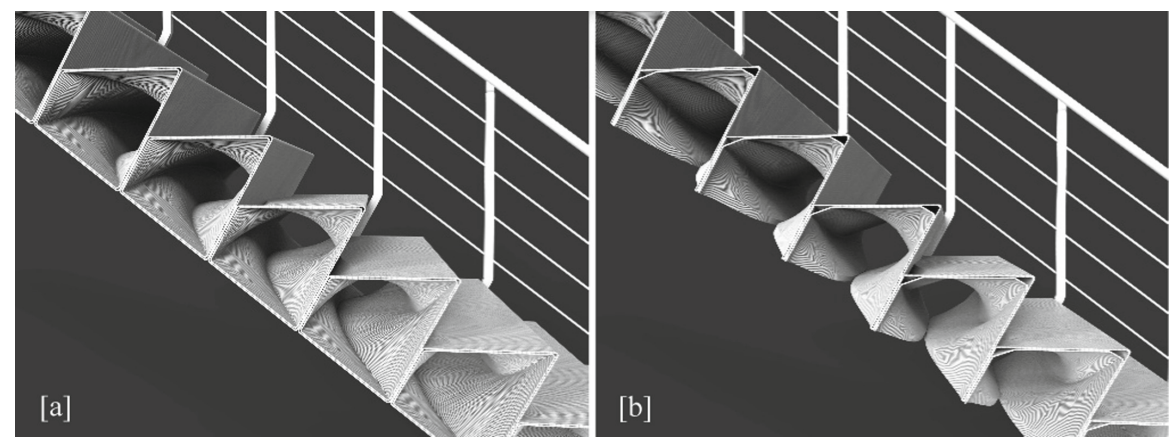

Fig. 2. Configuration 1, rendering of two versions of the module: Closed Shape (a) and open shape (b).

In the attempt of saving material, it has been developed a version without the surface opposite to the tread, leaving a connection between the riser and the back face only in the central part (Fig. 2b). This has been evaluated only in a scale model, showing potential for further investigations.
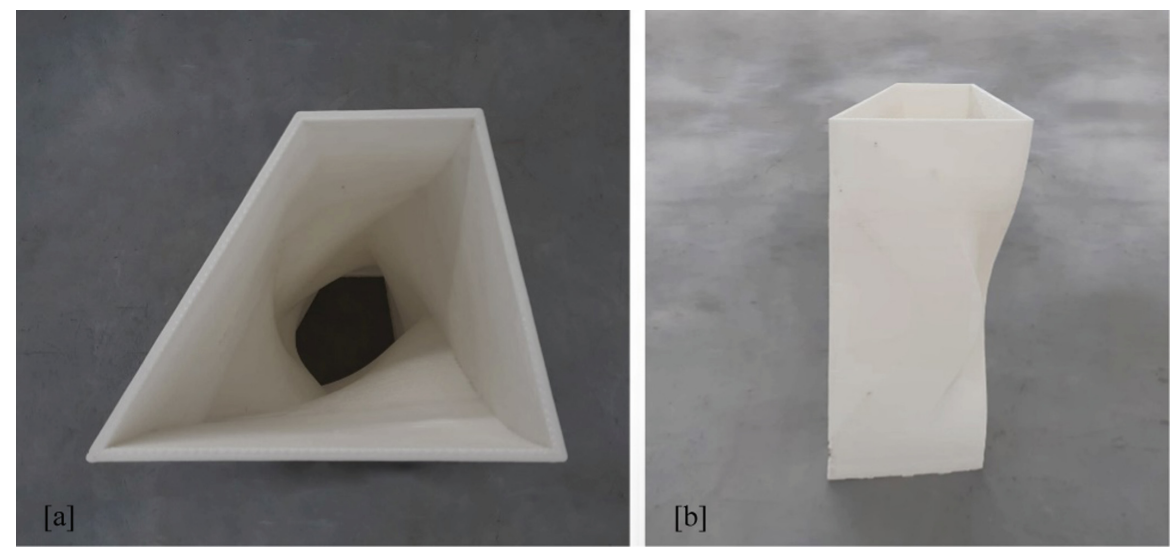

Fig. 3. Configuration 1, photos of the prototype. The module is printed in PLA with a $5 \mathrm{~mm}$ nozzle. The layer height is $1 \mathrm{~mm}$ for a total mass of $12.5 \mathrm{~kg}$. 


\section{Design Configuration 2: Horizontal Stacking}

The second configuration (Fig. 4) is based on the horizontal stacking of the components, relying on mechanical fasteners to allow the possibility of an easy assembly and disassembly (Fig. 5b). To maximize the mechanical performance of the module, the extremities are based on triangular shapes. The linear connection between the two sections is expanded on the back to form an arc. This arc defines the boundary surface of connection between the modules. The outer surface of the module is simply the interpolation of the rise, tread, and the curved surface of the back: the analysis of the sections reveals the transition from a triangle shape to a trapezoidal one in the middle sections. The inner surface traces the triangular shape on one side to then interpolate it to the riser profile on the opposite side (Fig. 5a). This allows having one side that is closed and the other open to insert the fastenings for the mechanical connections and the inspections. For the spiral version, the open side is the inner one, where the triangle is smaller. As in the previous case, the articulation of the inner surface is necessary to reinforce the structural integrity of the whole step. The flat surface of the tread is indeed larger and needs a strut to avoid flexions under payloads. The inner shell is so used to generate two twisting surfaces that are reinforcing the tread. The area between the two curves has a double layer, but the loads are distributed down to the base of the step (Fig. 5a).

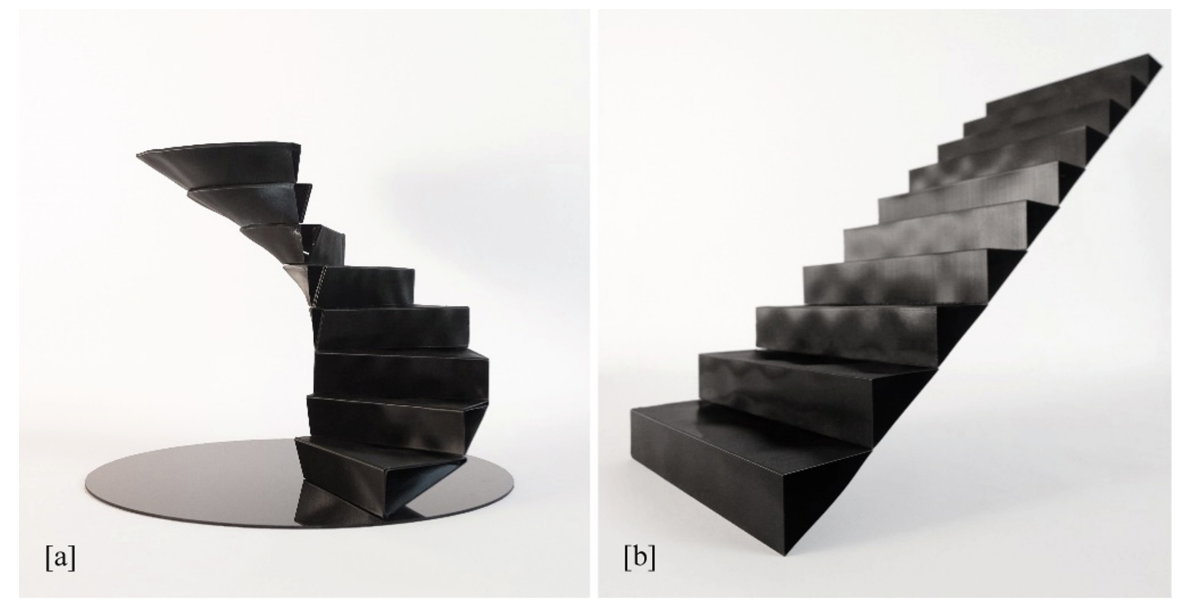

Fig. 4. Configuration 2, Photograph of the scale models. The module is developed in a spiral (a) and linear (b) version.

A series of steps have been produced with the printers manufactured by the Belgian company Colossus, using a carbon fiber $(10-<20 \%)$ filled recycled PETg $(80-<90 \%)$ material. The steps have been printed with a $5 \mathrm{~mm}$ nozzle and a layer height of $1,8 \mathrm{~mm}$. Printing a single step with that resolution takes between 4,5-5,5 h. The process is slowed down by the high resolution and precision required by the fact that is a final product. It must be considered that the printing speed is adjusted during the print process. The step is printed indeed, with a faster speed at the base where the shape is larger, and with a 


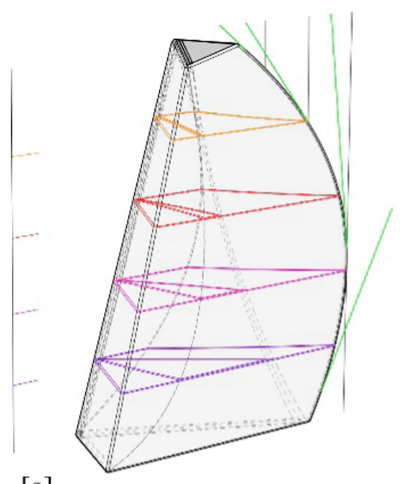

[a]

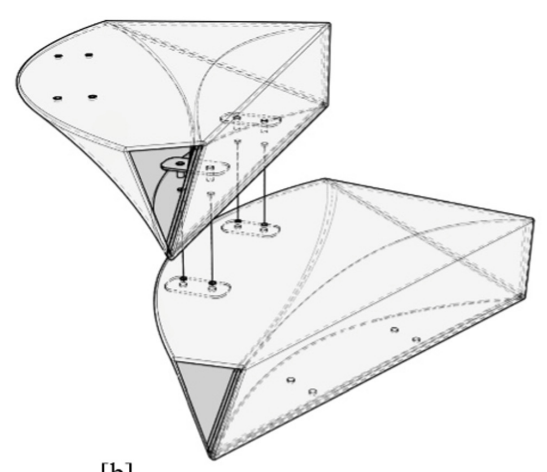

[b]

Fig. 5. Configuration 2, diagrams of the Spiral module. (a) Development of the section using the printing orientation. The design takes into consideration the limitation of the cantilever walls. (b) Assembly of the modules using mechanical fasteners.

slower speed at the top, where the contour lines are getting smaller. This is related to the temperature of the material and its solidification: if it doesn't slow down the extruded part remains too hot for the next layer, causing unwanted deformations. Particular care must be invested in the definitions of the edges. Sharp edges are difficult to print because the robot cannot change the direction of movement and simultaneously keep the printing speed constant. This is an advantage, anyway, because filleting the edges increases the solidity between the surfaces.

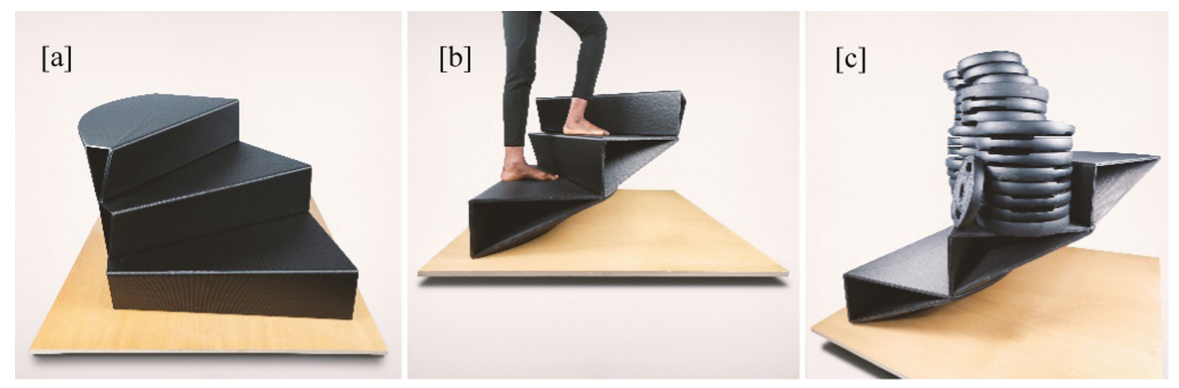

Fig. 6. Configuration 2, Photographs of three modules prototype. (a) Front, (b) Side, (c) $2452 \mathrm{~N}$ payload test.

The final mass of the single step is about $10.8 \mathrm{~kg}$. In the experience of the authors, the average mass for a custom staircase with a steel structure and metal sheet tread is between $17-30 \mathrm{~kg}$. An empirical series of tests have been done to verify the integrity of a single step under a distributed load of $5394 \mathrm{~N}$. Three steps mechanically fastened were also tested with a payload of $2452 \mathrm{~N}$ on the first, and the second step (Fig. 6c). The promising results of these initial verifications, opened the possibility for a more accurate series of testing to verify inflections and breaking load, and the testing of an entire staircase. 


\section{Conclusions and Further Development}

This paper proposes an approach to FGF design that balances process efficiency with a strong design focus, aiming to articulate a performative yet unique aesthetical quality for staircase design. The combination of BRep design and AM possess a wide range of architectural qualities that have to be explored and have the potentials to radically change the design and the construction processes so the language and identity of contemporary architecture, with functional, hollow structures.

After this first phase of testing that demonstrates the viability and sets a reference point, much further research has to be done on points like structural and mechanical stability, material life, fire resistance, the toxic effect of materials, etc. In particular, regarding the material and printing process, the opportunities are open to exploring other recycled materials, or bio-plastics. Another study concerns the adoption of TCM processes, using robotic-arm-based printing systems, especially in the case of spiral staircases. At last, new fabrication methods suggest also the definition of custom computational tools embedding the printing and material properties.

\section{References}

Al Jassmi, H., et al.: IOP Conference Series: Materials Science and Engineering, vol. 324, p. 012088 (2018)

Ashby, M.: Resource consumption and its drivers In: Materials and the Environment: Eco-Informed Material Choice. Butterworth-Heinemann, Amsterdam (2012)

Beyhan, F., Arslan, S.S.: 3D Printing in Architecture: One Step Closer to a Sustainable Built Environment (2018). https://doi.org/10.1007/978-3-319-63709-9_20

Celani, M.G., Mitchell, W., Knight, T.: Beyond analysis and representation in CAD: a new computational approach to design education. Massachusetts Institute of Technology (2002)

CSC Leading Edge Forum: 3D printing and the future of manufacturing (2012). http://assets1.csc. com/innovation/downloads/LEF_20123DPrinting.pdf. Accessed on 11 March 2021

Griffiths, S.: Giant 3D printer creates 10 full-sized houses in a DAY: Bungalows built from layers of waste materials cost less than $£ 3,000$ each. DailyMail (2014). http://www.dailymail.co.uk/sciencetech/article-2615076/Giant-3D-printer-creates-10sizedhouses-DAY-Bungalows-built-layers-waste-materials-cost-3-000-each.html. Accessed on 11 March 2021

Hitti, N.: Nagami's first collection features 3D-printed chairs by Zaha Hadid Architects, Dezeen (2018). https://www.dezeen.com/2018/03/26/nagamis-first-furniture-collectionfeatures-3d-printed-chairs-by-zaha-hadid-architects/. Accessed on 14 March 2021

Housing Observer: 3D printing and the construction industry (2015). https://www.yumpu.com/ en/document/read/54873923/housing-observer. Accessed on 11 March 2021

Jipa, A., et al.: 3D-printed formwork for bespoke concrete stairs: from computational design to digital fabrication, pp. 1-12 (2019). https://doi.org/10.1145/3328939.3329003

Khoshnevis, B.: Automated construction by contour crafting-related robotics and information technologies. Autom. Constr. 13(2004), 5-19 (2004)

Munoz, C., Kim, C., Armstrong, L.: Layer-by-layer: opportunities in 3D printing technology trends, growth drivers and the emergence of innovative applications in 3D printing. MaRS Mark Insights (2013). https://www.marsdd.com/wp-content/uploads/2014/04/MAR-CLT6965_3DPrinting_White_paper.pdf. Accessed on 11 March 2021

Reeves, P.: Additive Manufacturing - A supply chain wide response to economic uncertainty and environmental sustainability, Econolyst Limited, The Silversmiths, Derbyshire, UK (2009) 
Open Access This chapter is licensed under the terms of the Creative Commons Attribution 4.0 International License (http://creativecommons.org/licenses/by/4.0/), which permits use, sharing, adaptation, distribution and reproduction in any medium or format, as long as you give appropriate credit to the original author(s) and the source, provide a link to the Creative Commons license and indicate if changes were made.

The images or other third party material in this chapter are included in the chapter's Creative Commons license, unless indicated otherwise in a credit line to the material. If material is not included in the chapter's Creative Commons license and your intended use is not permitted by statutory regulation or exceeds the permitted use, you will need to obtain permission directly from the copyright holder. 